\title{
UNCOUNTABLE GROUPS AND THE GEOMETRY OF INVERSE LIMITS OF COVERINGS
}

\author{
GREGORY R. CONNER ${ }^{1}$, WOLFGANG HERFORT ${ }^{2}$, CURTIS KENT ${ }^{3}$, \\ AND PETAR PAVEŠIĆ ${ }^{4}$
}

\begin{abstract}
In this paper we develop a new approach to the study of uncountable fundamental groups by using Hurewicz fibrations with the unique path-lifting property (lifting spaces for short) as a replacement for covering spaces. In particular, we consider the inverse limit of a sequence of covering spaces of $X$. It is known that the path-connectivity of the inverse limit can be expressed by means of the derived inverse limit functor $\lim ^{1}$, which is, however, notoriously difficult to compute when the $\pi_{1}(\overleftarrow{X})$ is uncountable. To circumvent this difficulty, we express the set of path-components of the inverse limit, $\widetilde{X}$, in terms of the functors $\lim _{\mathrm{m}}$ and $\lim ^{1}$ applied to sequences of countable groups arising from polyhedral approximations of $X$.

A consequence of our computation is that path-connectedness of lifting space implies that $\pi_{1}(\widetilde{X})$ supplements $\pi_{1}(X)$ in $\check{\pi}_{1}(X)$ where $\check{\pi}_{1}(X)$ is the inverse limit of fundamental groups of polyhedral approximations of $X$. As an application we show that $\mathcal{G} \cdot \operatorname{Ker}_{\mathbb{Z}}(\widehat{F})=\widehat{F} \neq \mathcal{G} \cdot \operatorname{Ker}_{B(1, n)}(\widehat{F})$, where $\widehat{F}$ is the canonical inverse limit of finite rank free groups, $\mathcal{G}$ is the fundamental group of the Hawaiian Earring, and $\operatorname{Ker}_{A}(\widehat{F})$ is the intersection of kernels of homomorphisms from $\widehat{F}$ to $A$.
\end{abstract}

\section{INTRODUCTION}

A famous theorem of Shelah [27] states that the fundamental groups of Peano continua present a striking dichotomy: they are either finitely presented or uncountable. The first case corresponds to fundamental groups of finite polyhedra and covering space theory has traditionally been an effective geometric approach to the study of the structure of these groups. The second case is by no means exotic either: Peano continua with uncountable fundamental group arise naturally as attractors of dynamical systems [20, as fractal spaces [25, 29], as boundaries of non-positively curved groups [23],

Date: January 28, 2021.

${ }^{1}$ Supported by Simons Foundation Collaboration Grant 646221.

2 The second author is grateful for the warm hospitality at the Mathematics Department of BYU in February 2018.

${ }^{3}$ Supported by Simons Foundation Collaboration Grant 587001.

${ }^{4}$ Supported by the Slovenian Research Agency program P1-0292 and grants N1-0083, N1-0064. 
and in many other situations. The fundamental difference between the two cases is of a local nature. If the fundamental group of a Peano continuum $X$ is uncountable, then by [27] it is not semilocally simply connected at some point. As a consequence most of the subgroups of $\pi_{1}(X)$ do not correspond to a covering space over $X$, which represents a major obstacle for a geometric study of these groups. This work is part of a wider program to study fundamental groups of 'wild' Peano continua where the role of covering spaces is taken by more general fibrations with the unique path-lifting property. These fibrations were introduced by Spanier [28, Chapter 2] who developed much of the theory of covering spaces within this more general setting. Since the term 'Hurewicz fibration with the unique path-lifting property' is somewhat impractical we call them lifting spaces and the corresponding maps from the total space to the base are called lifting projections.

The main advantage of lifting spaces over covering spaces is that the former are closed with respect to composition and arbitrary inverse limits see [28, Section II.2]. Most notably, the inverse limit of a sequence of covering spaces over $X$ is always a lifting space over $X$ (and is not a covering projection, unless the sequence is eventually constant). If $X$ is semilocally simply-connected (e.g., a CW-complex), and the sequence is not eventually constant, then the limit lifting space is path-disconnected (see Corollary 2.2).

This is a geometric reflection of the fact that the fundamental group of the base is countable while the fibre of the lifting projection is uncountable. However, if $X$ is not semilocally simply-connected, then its fundamental group is uncountable by Shelah's theorem and the limit space can be pathconnected or not path-connected, depending on the interplay between $\pi_{1}(X)$ and the sequence of subgroups corresponding to the coverings in the inverse sequence.

The path-components of an inverse limit of covering spaces are classically determined by the derived inverse limit functor $\lim ^{1}$ applied to a sequence of subgroups of $\pi_{1}(X)$. Difficulties arise in the computation of $\lim ^{1}$ for inverse sequences of uncountable groups, which make this an ineffective approach to determining path-connectivity (see discussion at the end of Section 2 and Examples 3.7-3.11 in Section 3) . In Section 3, we consider inverse sequences of coverings over some polyhedral expansion of the base space $X$ and study the path-connectedness of the limit. We prove the following result, which allows one to work with inverse sequences of countable groups and thus avoid the difficulties of computing $\lim ^{1}$ for uncountable groups.

Theorem 1 (Theorem 3.4). Every inverse limit of covering projections over a Peano continuum $X$ is homeomorphic to an inverse limit of covering projections over a polyhedral expansion of $X$. 
The main result of Section 3, Theorem 3.5, completely describes the fundamental group and the set of path components of an inverse limit of coverings over some polyhedral expansions of a Peano continuum. The statement is quite technical, but it leads to the following important consequence which characterizes path-connectedness of the limit space.

Corollary 2 (Corollary 3.6). Let $p: \widetilde{X} \rightarrow X$ be the inverse limit of covering maps over a Peano continuum $X$. Then $\widetilde{X}$ is path-connected if, only if, the inverse sequence of fundamental groups, $\pi_{1}\left(\widetilde{X}_{i}\right)$, satisfies the Mittag-Leffler property and the natural homomorphism

$$
\pi_{1}(X) \longrightarrow \lim _{\longleftarrow}\left(\frac{\pi_{1}\left(X_{i}\right)}{\pi_{1}\left(\widetilde{X}_{i}\right)}\right)
$$

is surjective, where $\tilde{X}_{i} \rightarrow X_{i}$ are covering projections of a polyhedral expansion $\left\{X_{i}\right\}$ of $X$.

Even for the well-studied Hawaiian earring group $\mathcal{G}$, apart from the fact that $\mathcal{G}$ contains the free group of countable rank and is thus dense in its shape group $\widehat{F}$, very little is known about the size of $\mathcal{G}$ in $\widehat{F}$. The above corollary leads to interesting algebraic applications concerning the image of the fundamental group into the shape group which we consider in Section 4.

Given groups $G$ and $A$, we let $\operatorname{Ker}_{A}(G)$ be the intersection of kernels of all homomorphisms from $G$ to $A$. If $G$ is a free group and $B(1, n)$ is a Baumslag-Solitar group, then $\operatorname{Ker}_{\mathbb{Z}}(G)$ is exactly the commutator subgroup of $G$ and $\operatorname{Ker}_{B(1, n)}(G)$ is exactly the second derived subgroup of $G$, see [11]. However, $\widehat{F}$ is locally free but non-free and the corresponding $\operatorname{Ker}_{\mathbb{Z}}(\widehat{F})$ properly contains the commutator subgroup of $\widehat{F}$. In fact, $\operatorname{Ker}_{\mathbb{Z}}(\widehat{F})$ is sufficiently large to be a supplement of $\mathcal{G}$ in $\widehat{F}$ while $\operatorname{Ker}_{B(1, n)}(\widehat{F})$ is not.

Theorem 3 (Theorem 4.4 and Theorem 4.5). The group $\widehat{F}$ is equal to the internal product of its subgroups $\mathcal{G}$ and $\operatorname{Ker}_{\mathbb{Z}}(\widehat{F})$, while the internal product of $\mathcal{G}$ and $\operatorname{Ker}_{B(1, n)}(\widehat{F})$ is a proper subgroup of $\widehat{F}$.

\section{Preliminaries on inverse limits of SPACES AND Groups}

The main object of our study are lifting spaces that arise as inverse limits of covering spaces, so let us consider the following sequence of regular covering maps $p_{i}: \widetilde{X}_{i} \rightarrow X$, together with the inverse limit map $p: \widetilde{X} \rightarrow X$ :

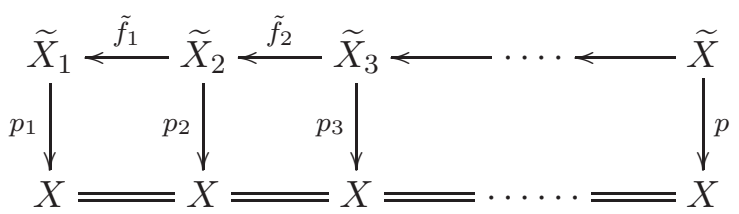


For each $i$ we identify $\pi_{1}\left(\widetilde{X}_{i}\right)$ with its image $p_{i *}\left(\pi_{1}\left(\widetilde{X}_{i}\right)\right)$ which is a normal subgroup of $\pi_{1}(X)$. By standard covering space theory the bonding maps $\tilde{f}_{i}: \widetilde{X}_{i+1} \rightarrow \widetilde{X}_{i}$ are covering maps and we have a decreasing sequence of normal subgroups of $\pi_{1}(X)$

$$
\pi_{1}(X) \unrhd \pi_{1}\left(\widetilde{X}_{1}\right) \unrhd \pi_{1}\left(\widetilde{X}_{2}\right) \unrhd \cdots
$$

Note that the converse is not true for general spaces, because a subgroup of $\pi_{1}(X)$ may not be a covering subgroup, i.e. it does not necessarily come from a covering of $X$. However, if $X$ is locally path-connected, then by 28, Theorem II, 5.12] every decreasing sequence of normal covering subgroups of $\pi_{1}(X)$ uniquely determines an inverse sequence of regular coverings over $X$.

Let $F(p)$ denote the fibre of the lifting projection $p$. Then $F(p)$ is the inverse limit of the fibres $F\left(p_{i}\right)$, and so by [28, Theorem II, 6.2] it can be naturally identified with the inverse limit of the quotients

$$
F(p)=\lim _{\longleftarrow}\left(\pi_{1}(X) / \pi_{1}\left(\widetilde{X}_{i}\right)\right) .
$$

Since all bonding maps in the inverse sequence are fibrations, it is wellknown (see [4, 22]) that the homotopy groups of $\pi_{1}(\widetilde{X})$ can be expressed in terms of the homotopy groups of $\widetilde{X}_{i}$ :

$$
\pi_{1}(\tilde{X})=\lim _{\longleftarrow} \pi_{1}\left(\widetilde{X}_{i}\right)=\bigcap_{i} \pi_{1}\left(\widetilde{X}_{i}\right), \quad \pi_{0}(\widetilde{X})=\lim ^{1} \pi_{1}\left(\widetilde{X}_{i}\right)
$$

and $\pi_{n}(\tilde{X})=\pi_{n}(X)$ for $n>1$. Here $\lim _{\longleftarrow}$ denotes the inverse limit functor on groups and $\lim ^{1}$ is its first derived functor (see [19, Section 11.3]). For commutative groups these functors can be defined as kernel and cokernel of the homomorphism $\varphi: \prod \pi_{1}\left(\widetilde{X}_{i}\right) \rightarrow \prod \pi_{1}\left(\widetilde{X}_{i}\right)$, given as

$$
\varphi\left(u_{1}, u_{2}, u_{3}, \ldots\right)=\left(u_{1}-\tilde{f}_{1 *}\left(u_{2}\right), u_{2}-\tilde{f}_{2 *}\left(u_{3}\right), \ldots\right)
$$

and so they fit in the exact sequence

$$
0 \rightarrow \lim _{\longleftarrow} \pi_{1}\left(\tilde{X}_{i}\right) \rightarrow \prod \pi_{1}\left(\widetilde{X}_{i}\right) \stackrel{\varphi}{\longrightarrow} \prod \pi_{1}\left(\widetilde{X}_{i}\right) \rightarrow \lim ^{1} \pi_{1}\left(\widetilde{X}_{i}\right) \rightarrow 0 .
$$

The non-commutative case is more delicate: in that case $\lim ^{1} \pi_{1}\left(\widetilde{X}_{i}\right)$ is defined as the quotient of $\prod \pi_{1}\left(\widetilde{X}_{i}\right)$ under the equivalence relation given as

$$
\left(u_{i}\right) \sim\left(v_{i}\right) \Leftrightarrow\left(v_{i}\right)=\left(x_{i} u_{i} \tilde{f}_{i *}\left(x_{i+1}\right)^{-1}\right) \text { for some }\left(x_{i}\right) \in \prod \pi_{1}\left(\tilde{X}_{i}\right)
$$

(see [17] for detailed treatment of the non-commutative case).

The values of $\lim ^{1}$ are notoriously hard to compute. Here we will be only interested whether $\lim ^{1}$ of a sequence is trivial, and this can be settled if we can show that the inverse sequence satisfies the Mittag-Leffler condition, which we now define. In an inverse sequence of groups

$$
G_{1} \longleftarrow G_{2} \longleftarrow G_{3} \longleftarrow \cdots
$$


for a fixed $j$ the image of the homomorphism $G_{i} \rightarrow G_{j}$ decreases as $i$ goes toward infinity. The inverse sequence is said to satisfy the Mittag-Leffler condition if for every $j$ the sequence $\left\{\operatorname{Im}\left(G_{i} \rightarrow G_{j}\right) \mid i=j, j+1, \ldots\right\}$ stabilizes. Clearly, sequences with epimorphic bonding maps satisfy the Mittag-Leffler condition. On the other hand, if the bonding maps are monomorphisms, then the sequence satisfies he Mittag-Leffler condition if, and only if, it is eventually constant. The following result is proved in 17] (see also [19, Theorem 11.3.2]):

Proposition 2.1. If an inverse sequence $\left\{G_{i}\right\}$, of groups, satisfies the Mittag-Leffler condition, then $\lim ^{1} G_{i}=\{1\}$. Conversely, if $\lim ^{1} G_{i}=\{1\}$ and each $G_{i}$ is countable, then $\left.\overleftarrow{\{G}_{i}\right\}$ satisfies the Mittag-Leffler condition.

As we explained before, the sequence of fundamental groups induced by an inverse sequence of coverings satisfies Mittag-Leffler condition only if it is constant from some point on. Thus we get immediately the following corollary.

Corollary 2.2. Let $\widetilde{X}$ be the inverse limit of an inverse sequence of covering maps $p_{i}: \widetilde{X}_{i} \rightarrow X$ and assume that for some $i$ the group $\pi_{1}\left(\widetilde{X}_{i}\right)$ is countable. Then either the sequence is eventually constant (which implies that $p: \widetilde{X} \rightarrow$ $X$ is a covering map), or $\widetilde{X}$ is not path-connected.

In particular an inverse limit of coverings over a countable $C W$-complex is either a covering or its total space is not path-connected.

This last statement is not surprising if one considers the tail of the exact homotopy sequence of the fibration $p: \widetilde{X} \rightarrow X$, shown in the following diagram together with the above mentioned identifications:

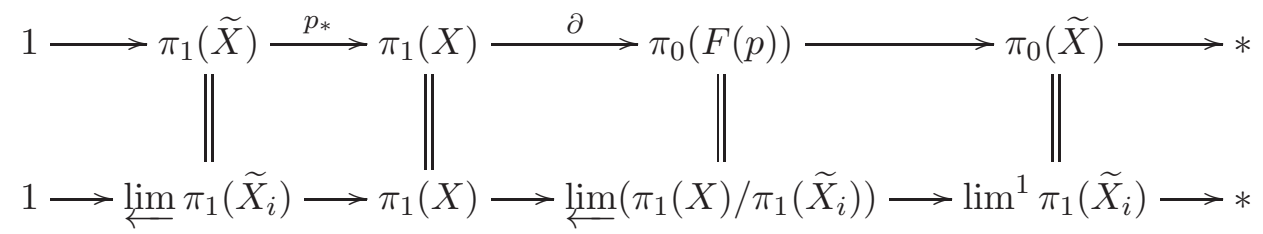

If the sequence is not eventually constant, then $\pi_{0}(F(p))$ is uncountable. But if $X$ is a countable CW-complex, then $\pi_{1}(X)$ is a countable group and so $\partial$ cannot be surjective.

However, if $\pi_{1}(X)$ is uncountable, then $\partial$ can be surjective. For example, the lifting projection over the infinite product of circles, obtained by 'unwrapping' one circle at a time, as in

$$
\left(S^{1}\right)^{\infty} \leftarrow\left(\mathbb{R} \times S^{1} \times S^{1} \times \ldots\right) \leftarrow\left(\mathbb{R} \times \mathbb{R} \times S^{1} \times \ldots\right) \leftarrow \cdots \cdots \leftarrow \mathbb{R}^{\infty}
$$

has a path-connected total space in spite of the fact that it is not eventually constant. Another, less obvious example is a sequence of 2 -fold coverings 
over the Hawaiian earring whose limit space is path-connected (see [9, Section 2.7]). In both cases we have decreasing sequences of uncountable fundamental groups that do not satisfy the Mittag-Leffler condition but their derived inverse limits are nonetheless trivial.

Of course, one can easily find examples of inverse limits of coverings of the Hawaiian ring whose total space has uncountably many path-components. Distinguishing between different cases is hindered by the difficulties in the explicit computation of the derived limit functor for decreasing sequences of uncountable groups. We present one approach to this problem in the following section, while in the last section we consider the algebraic implications of the path-(dis)connectedness of the limit space.

\section{InVERSE LIMITS OF COVERINGS OVER AN EXPANSION OF $X$}

Our approach to reducing the complexity of computing the derived limit functor to the more manageable countable case is to consider a polyhedral expansion $\left\{X_{i}\right\}$ of $X$ and represent a lifting projection $p: \widetilde{X} \rightarrow X$ as an inverse limit of covering maps over the polyhedral expansion, i.e., find covering maps $p_{i}: \widetilde{X}_{i} \rightarrow X_{i}$ that satisfy the following diagram.

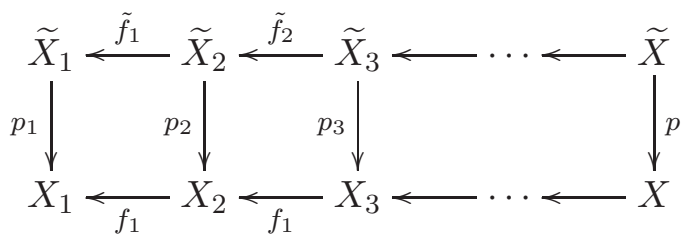

There are several standard methods by which a compact metric space can be represented as a limit of an inverse sequence of polyhedra (see MardesicSegal [26]). The one that best suits our purposes is by nerves of coverings, which we briefly recall (see [26, Appendix 1] for details). Every metric compactum $X$ admits arbitrarily fine finite open coverings. Moreover, for every finite open covering $\mathcal{U}=\left\{U_{1}, \ldots, U_{n}\right\}$ of $X$ there exists a subordinated partition of unity $\left\{\rho_{i}: X \rightarrow[0,1] \mid i=1, \ldots, n\right\}$. Let $N(\mathcal{U})$ be the nerve of the covering $\mathcal{U}$, i.e., the simplicial complex whose vertices are elements of $\mathcal{U}$, and whose simplices are spanned by elements of $\mathcal{U}$ with non-empty intersection. Then the formula

$$
f(x):=\sum_{i=1}^{n} \rho_{i}(x) \cdot U_{i}
$$

defines a map $f: X \rightarrow|N(\mathcal{U})|$ (where $|N(\mathcal{U})|$ is the geometric realization of the nerve). It is well known that the choice of the partition of unity does not affect the homotopy class of $f$, so the induced homomorphism $f_{*}: \pi_{1}(X) \rightarrow \pi_{1}(|N(\mathcal{U})|)$ depends only on the cover $\mathcal{U}$. 
Lemma 3.1. If each element of $\mathcal{U}$ is path-connected, then $f_{*}: \pi_{1}(X) \rightarrow$ $\pi_{1}(|N(\mathcal{U})|)$ is surjective.

Proof. Without loss of generality we may assume that the partition of unity subordinated to $\mathcal{U}$ is reduced in the sense that for every $i$ there exists $x_{i} \in U_{i}$, such that $\rho_{i}\left(x_{i}\right)=1$. For every pair of intersecting sets $U_{i}, U_{j} \in \mathcal{U}$ we may choose a path in $U_{i} \cup V_{j}$ between $x_{i}$ and $x_{j}$. These paths determine a map $g:\left|N(\mathcal{U})^{(1)}\right| \rightarrow X$ from the 1-skeleton of the nerve to $X$. One can check that, for every 1-simplex $\sigma$ in $N(\mathcal{U})^{(1)}$, the image $f(g(\sigma))$ is contained in the open star of $\sigma$ in $N(\mathcal{U})$, which implies that $f \circ g$ is homotopic to the inclusion $i:\left|N(\mathcal{U})^{(1)}\right| \hookrightarrow|N(\mathcal{U})|$. Since $i_{*}: \pi_{1}\left(\left|N(\mathcal{U})^{(1)}\right|\right) \rightarrow \pi_{1}(|N(\mathcal{U})|)$ is surjective, $f_{*}$ is also surjective.

If $\mathcal{U}^{\prime}$ is a covering of $X$ that refines $\mathcal{U}$ (i.e. every element of $\mathcal{U}^{\prime}$ is contained in some element of $\mathcal{U})$, then there is an obvious simplicial map $N\left(\mathcal{U}^{\prime}\right) \rightarrow N(\mathcal{U})$. By iterating the refinements we obtain an inverse system of polyhedra

$$
\left.\left.\left.\mid N\left(\mathcal{U}_{1}\right)\right)|\longleftarrow| N\left(\mathcal{U}_{2}\right)\right)|\longleftarrow| N\left(\mathcal{U}_{3}\right)\right) \mid \longleftarrow \ldots
$$

whose limit is $X$. Moreover, if $X$ is locally path-connected, then we may choose covers of $X$ whose elements are path-connected. As an interesting aside, Lemma 3.1 immediately implies the following well-known fact, which also follows immediately from the Hahn-Mazurkiewicz theorem and results of Krasinkiewicz [24, Theorems 4.1 and 4.2].

Corollary 3.2. Every Peano continuum $X$ can be represented as the limit of an inverse system of finite polyhedra

$$
X_{1} \longleftarrow X_{2} \longleftarrow X_{3} \longleftarrow \cdots \longleftarrow \lim _{\longleftarrow} X_{i}=X
$$

such that the homomorphisms $\pi_{1}(X) \rightarrow \pi_{1}\left(X_{i}\right)$ induced by the projection maps are surjective.

We do not know whether every inverse limit of coverings over a pathconnected and locally path-connected base $X$ can be derived from an inverse limit of coverings over some expansion of $X$. However, if we assume that $X$ is compact, we may use the approximation by nerves of coverings to prove the following theorem.

Lemma 3.3. Let $X$ be a Peano continuum and $g: X \rightarrow Y$ be a continuous map into a polyhedron $Y$. If $\mathcal{U}$ is a cover of $X$ by path connected open sets such that the preimage of the open star of any vertex of $Y$ is contained in an element of $\mathcal{U}$, then $\operatorname{Ker}\left(g_{*}\right) \subset \pi_{1}(X ; 2 \mathcal{U})$.

Proof. If we denote by $f: X \rightarrow|\mathcal{U}|$ the map from $X$ to the realization of the nerve of $\mathcal{U}$, then the kernel of the homomorphism $f_{*}: \pi_{1}(X) \rightarrow \pi_{1}(|\mathcal{U}|)$ is $\pi_{1}(X ; 2 \mathcal{U})$, see [8, Lemma 3.3]. Since the preimage of the open star of any vertex of $Y$ is contained in an element of $\mathcal{U}$, there exists a map $h: Y \rightarrow|\mathcal{U}|$ such that $f_{*}=h_{*} \circ g_{*}$. Thus $\operatorname{Ker}\left(g_{*}\right) \subset \operatorname{ker}\left(f_{*}\right)=\pi_{1}(X ; 2 \mathcal{U})$. 
Let $\mathcal{U}$ be an open cover of $X$. We will use $\pi_{1}(X ; \mathcal{U})$ to denote the $\mathcal{U}$-Spanier subgroup, i.e. $\pi_{1}(X ; \mathcal{U})$ is the subgroup of $\pi_{1}\left(X, x_{0}\right)$ generated by

$$
\left\{[\alpha * \beta * \bar{\alpha}] \mid \alpha:(I, 0) \rightarrow\left(X, x_{0}\right) \text { and } \operatorname{Im}(\beta) \subset U \text { for some } U \in \mathcal{U}\right\} .
$$

Theorem 3.4. If $X$ is a Peano continuum, then every inverse sequence of covering projections over $X$ is homeomorphic to a pull-back of an inverse sequence of covering maps over a given polyhedral expansion of $X$.

As a consequence, every inverse limit of covering maps over $X$ is homeomorphic to an inverse limit of covering maps over a given polyhedral expansion of $X$.

Proof. Let

$$
X_{1} \longleftarrow X_{2} \longleftarrow X_{3} \longleftarrow \cdots \longleftarrow \lim _{\longleftarrow} X_{i}=X
$$

be a polyhedral expansion of $X$, such that the homomorphisms $\pi_{1}(X) \rightarrow$ $\pi_{1}\left(X_{i}\right)$ are surjective.

Let $p: \widetilde{X} \rightarrow X$ be an arbitrary covering map. Then $p$ evenly covers elements of some open cover $\mathcal{U}$ of $X$ and $\pi_{1}(\widetilde{X}) \supseteq \pi_{1}(X ; \mathcal{U})$, where the latter denotes the $\mathcal{U}$-Spanier subgroup of $\pi_{1}(X)$. Since $X$ is compact we may assume that $\mathcal{U}$ is finite. Choose another finite cover $\mathcal{V}$ of $X$ by path connected open sets whose double $2 \mathcal{V}$ (set of all unions of two elements of $\mathcal{V}$ ) refines $\mathcal{U}$.

By Theorem 5 of [26] there exists an $i$ such that the preimage of the open star of any vertex of $X_{i}$ is contained in an element of $\mathcal{V}$. Then by Lemma 3.3. we have

$$
\pi_{1}(\tilde{X}) \supseteq \pi_{1}(X ; \mathcal{U}) \supseteq \pi_{1}(X ; 2 \mathcal{V}) \supseteq \operatorname{Ker}\left(\pi_{1}(X) \rightarrow \pi_{1}\left(X_{i}\right)\right) .
$$

Therefore, if

$$
X \longleftarrow \widetilde{X}_{1} \longleftarrow \widetilde{X}_{2} \longleftarrow \widetilde{X}_{3} \longleftarrow \cdots
$$

is an inverse sequence of coverings over $X$ we may find for each $i$ some index $j(i)$ such that $\pi_{1}\left(\widetilde{X}_{i}\right) \supseteq \operatorname{Ker}\left(\pi_{1}(X) \rightarrow \pi_{1}\left(X_{j(i)}\right)\right)$.

Denote $K_{i}:=\operatorname{Ker}\left(\pi_{1}(X) \rightarrow \pi_{1}\left(X_{j(i)}\right)\right)$ and let $\bar{X}_{j(i)} \rightarrow X_{j(i)}$ be the covering map that corresponds to the subgroup $\operatorname{Im}\left(\pi_{1}\left(\widetilde{X}_{i}\right) \rightarrow \pi_{1}\left(X_{j(i)}\right)\right)$. By the lifting theorem, there is a fibre-preserving map $\tilde{f}_{i}: \widetilde{X}_{i} \rightarrow \bar{X}_{j(i)}$ for which the following diagram commutes

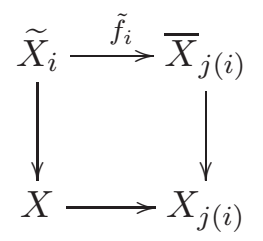


To compute the restriction of $\tilde{f}_{i}$ on the fibres we examine the following commutative diagram with exact rows

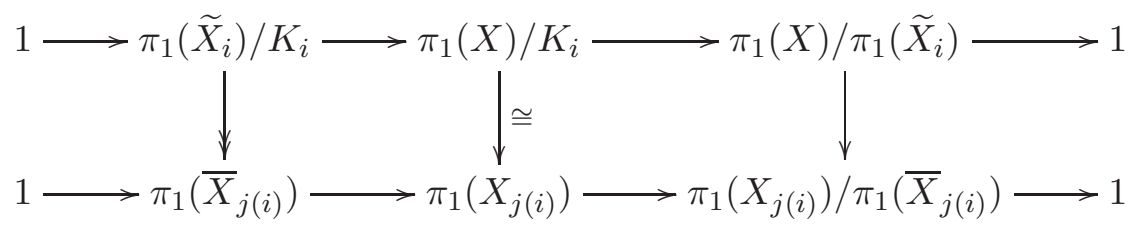

By the short five-lemma we conclude that $\tilde{f}_{i}$ induces an isomorphism

$$
\pi_{1}(X) / \pi_{1}\left(\tilde{X}_{i}\right) \rightarrow \pi_{1}\left(X_{j(i)}\right) / \pi_{1}\left(\bar{X}_{j(i)}\right)
$$

and hence a bijection between the fibres of respective covering maps. In other words, the above diagram is a pull-back of covering maps.

By repeating the above argument for all terms in an inverse sequence of covering maps over $X$ we obtain an inverse sequence of covering maps over the given expansion of $X$ with the same limit lifting projection.

The above theorem shows that for most cases of interest it is sufficient to consider lifting projections over $X$ that are inverse limits of covering maps over some expansion of $X$ as in

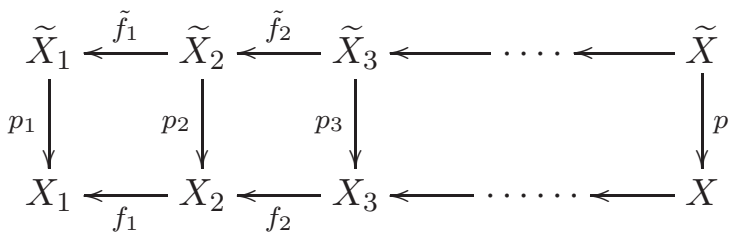

Suppose that $X_{i}$ are polyhedra and that the maps $X \rightarrow X_{i}$ induce epimorphisms between respective fundamental groups (e.g. if $X$ is locally path-connected). As in Section 2, we may compare the tail of the exact homotopy sequence of the fibration $p$ with the derived exact sequence of the inverse limit functor to obtain the following diagram

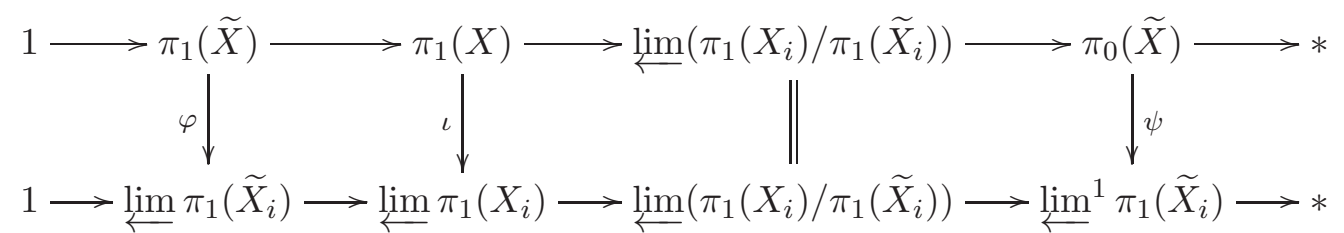

The inverse limits $\lim _{\longleftarrow} \pi_{1}\left(X_{i}\right)$ and $\lim \pi_{1}\left(\widetilde{X}_{i}\right)$ are independent of the choice of a polyhedral expansion for $X$. That two polyhedral expansions give proisomorphic inverse limit groups is proved in Mardesic-Segal [26, Ch 2] and the pro-isomorphism can be lifted to a pro-isomorphism of the corresponding covering subgroups via the homotopy lifting criterion. The limit $\lim _{\longleftarrow} \pi_{1}\left(X_{i}\right)$ is usually called the $\breve{C} e c h$ fundamental group (or the first shape group) of $X$, and is denoted by $\check{\pi}_{1}(X)$. Since $\widetilde{X}$ is not necessarily compact, $\lim \pi_{1}\left(\widetilde{X}_{i}\right)$ is 
not necessarily the first shape group of $\widetilde{X}$. Regardless, we will still denote $\lim _{1}\left(\widetilde{X}_{i}\right)$ by $\check{\pi}_{1}(\widetilde{X})$. The kernel of the induced map $\iota: \pi_{1}(X) \rightarrow \check{\pi}_{1}(X)$ is called the shape kernel of $X$ and denoted $\operatorname{ShKer}(X)$. Note that by the exactness of the above diagram we have that

$$
\operatorname{Ker}\left(\pi_{1}(\tilde{X}) \rightarrow \lim _{\longleftarrow} \pi_{1}\left(\tilde{X}_{i}\right)\right)=\operatorname{Ker}\left(\pi_{1}(X) \rightarrow \lim _{\longleftarrow} \pi_{1}\left(X_{i}\right)\right)=\operatorname{ShKer}(X) .
$$

Clearly, $\psi: \pi_{0}(\widetilde{X}) \rightarrow \lim ^{1} \pi_{1}\left(\widetilde{X}_{i}\right)$ is a surjective function between homogeneous sets. Thus, in order to determine $\pi_{0}(\widetilde{X})$ we need to compute the fibres of $\psi$. From the exactness of the second row we deduce that $\lim ^{1} \pi_{1}\left(\widetilde{X}_{i}\right)$ is the set of cosets of the action of the group $\check{\pi}_{1}(X) / \check{\pi}_{1}(\widetilde{X})$ on $\lim \left(\pi_{1}\left(X_{i}\right) / \pi_{1}\left(\tilde{X}_{i}\right)\right)$. Then a straightforward diagram chasing shows that the fibres of $\psi$ can be naturally identified with the cosets of the action of $\iota\left(\pi_{1}(X)\right)$ on $\check{\pi}_{1}(X) / \check{\pi}_{1}(\tilde{X})$.

Theorem 3.5. Let $X$ be a path-connected space that admits a polyhedral expansion $f_{i}: X \rightarrow X_{i}$ such that the induced homomorphisms $f_{i *}$ are surjective and let $p: \widetilde{X} \rightarrow X$ be the inverse limit of a sequence of coverings $p_{i}: \widetilde{X}_{i} \rightarrow X_{i}$ as described above. Then the fundamental group of $\widetilde{X}$ is determined by the exact sequence of groups

$$
1 \rightarrow \operatorname{ShKer}(X) \rightarrow \pi_{1}(\widetilde{X}) \rightarrow \check{\pi}_{1}(\widetilde{X}),
$$

and the set of path-components of $\widetilde{X}$ is determined by the exact sequence of groups and based homogeneous sets

$$
\pi_{1}(X) \stackrel{\iota}{\longrightarrow} \check{\pi}_{1}(X) / \check{\pi}_{1}(\tilde{X}) \longrightarrow \pi_{0}(\tilde{X}) \longrightarrow \lim ^{1} \pi_{1}\left(\widetilde{X}_{i}\right) \longrightarrow *
$$

The main advantage of Theorem 3.5 with respect to the description of $\pi_{0}(\widetilde{X})$ given in Section 2 is that all limits and derived limits are taken over inverse sequences of countable groups.

Corollary 3.6. Let $p: \widetilde{X} \rightarrow X$ be the inverse limit of covering maps as in the above theorem. Then $\widetilde{X}$ is path-connected if, only if, the inverse sequence

$$
\pi_{1}\left(\widetilde{X}_{1}\right) \leftarrow \pi_{1}\left(\widetilde{X}_{2}\right) \leftarrow \pi_{1}\left(\widetilde{X}_{3}\right) \leftarrow \cdots
$$

satisfies the Mittag-Leffler property and the natural homomorphism

$$
\pi_{1}(X) \longrightarrow \frac{\check{\pi}_{1}(X)}{\check{\pi}_{1}(\widetilde{X})} \cong \lim _{\longleftarrow}\left(\frac{\pi_{1}\left(X_{i}\right)}{\pi_{1}\left(\widetilde{X}_{i}\right)}\right)
$$

is surjective.

Proof. Theorem 3.5 implies that $\pi_{0}(\tilde{X})$ is trivial if, and only if $\lim ^{1} \pi_{1}\left(\widetilde{X}_{i}\right)$ is trivial and $\pi_{1}(X) \longrightarrow \check{\pi}_{1}(X) / \check{\pi}_{1}(\tilde{X})$ is surjective. Since the fundamental groups of polyhedra are countable, then by Proposition 2.1 the inverse sequence is Mittag-Leffler if and only if $\lim ^{1} \pi_{1}\left(\widetilde{X}_{i}\right)$ is trivial. 
Observe that the isomorphism between $\check{\pi}_{1}(X) / \check{\pi}_{1}(\widetilde{X})$ and $\lim \left(\pi_{1}\left(X_{i}\right) / \pi_{1}\left(\widetilde{X}_{i}\right)\right)$ follows form the Mittag-Leffler property and does not hold in general.

Let us consider a couple of examples.

Example 3.7. We have already mentioned the inverse sequence of covering maps over the infinite product of circles

$$
\left(S^{1}\right)^{\infty} \leftarrow\left(\mathbb{R} \times S^{1} \times S^{1} \times \ldots\right) \leftarrow\left(\mathbb{R} \times \mathbb{R} \times S^{1} \times \ldots\right) \leftarrow \cdots
$$

It can be replaced by the following inverse sequence of covering maps over polyhedral approximations of $\left(S^{1}\right)^{\infty}$ (with horizontal maps the usual projections)

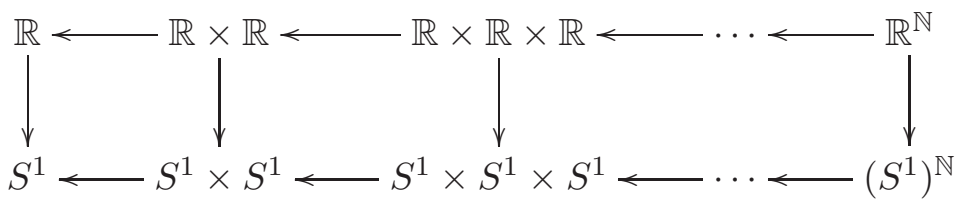

The fundamental groups of the products of copies of $\mathbb{R}$ are trivial so the corresponding sequence is Mittag-Leffler. By Corollary 3.6 the path-connectedness of $\mathbb{R}^{\mathbb{N}}$ is equivalent to the equality $\pi_{1}\left(\left(S^{1}\right)^{\mathbb{N}}\right)=\lim _{\longleftarrow} \pi_{1}\left(\left(S^{1}\right)^{n}\right)=\check{\pi}_{1}\left(\left(S^{1}\right)^{\mathbb{N}}\right)$.

Example 3.8. Let $T^{\infty}$ be the infinite product of circles endowed with the $\mathrm{CW}$-topology, i.e. the direct limit of the sequence of spaces

$$
S^{1} \hookrightarrow S^{1} \times S^{1} \hookrightarrow S^{1} \times S^{1} \times S^{1} \hookrightarrow \cdots T^{\infty}
$$

Clearly, the fundamental group of $T^{\infty}$ is $\bigoplus_{k=1}^{\infty} \mathbb{Z}$. For each $i \in \mathbb{N}$ let $p_{i}: \widetilde{X}_{i} \rightarrow T^{\infty}$ be the covering map that corresponds to the subgroup $\pi_{1}\left(\widetilde{X}_{i}\right)=$ $\bigoplus_{k=i}^{\infty} \mathbb{Z}$. By Corollary 2.2 the inverse limit $\widetilde{X}:=\lim _{\longleftarrow} \widetilde{X}_{i}$ is not pathconnected.

Remark 3.9. The last result is somewhat counter-intuitive, as one could argue that $\widetilde{X}_{i} \approx \mathbb{R}^{i-1} \times T^{\infty}$ and that the inverse limit of the coverings is simply the product $\mathbb{R}^{\infty}$ but the computation reveals that the geometry of the inverse limit must be different. We leave this as an (easy) exercise for the reader. As a hint, note that the fibre of $\widetilde{X} \rightarrow T^{\infty}$ is uncountable while the fundamental group of $T^{\infty}$ is countable.

Example 3.10. Consider the squaring lifting projection $p: \widetilde{H}_{\infty} \rightarrow H$ over the Hawaiian earring $H$ described in [9, Section 2.7]. The map $p$ is obtained as a limit of a sequence of 2-fold covering projections and, although it resembles at first sight the construction of the dyadic solenoid, we have been able to prove by a geometric argument that its total space $\widetilde{H}_{\infty}$ is pathconnected. We are going to show how this result is reflected in the algebraic computation of the set of path-components of $\widetilde{H}_{\infty}$.

As explained in [9, Section 3.3] we can obtain the projection $p$ as follows. Let $H_{i}$ be a wedge of $i$ circles, so that $H_{1} \leftarrow H_{2} \leftarrow \ldots \leftarrow H$ is the standard 
polyhedral expansion of the Hawaiian earring. For each $i$ let $p_{i}: \widetilde{H}_{i} \rightarrow H_{i}$ be the $2^{i}$-fold covering projection, obtained as the restriction to $H_{i} \subset\left(S^{1}\right)^{i}$ of the squaring map

$$
\left(S^{1}\right)^{i} \rightarrow\left(S^{1}\right)^{i}, \quad\left(z_{1}, \ldots, z_{i}\right) \mapsto\left(z_{1}^{2}, \ldots, z_{i}^{2}\right) .
$$

It is then easy to check that $p$ is the inverse limit of the sequence of coverings $p_{i}$, in particular $\widetilde{H}_{\infty}=\lim _{\longleftarrow} \widetilde{H}_{i}$.

The sequence of groups $\left(\pi_{1}\left(\widetilde{H}_{i}\right)\right)$ has surjective bonding maps, so by Corollary 3.6 the path-connectedness of $\widetilde{H}_{\infty}$ is equivalent to the surjectivity of the homomorphism $\pi_{1}(H) \rightarrow\left(\mathbb{Z}_{2}\right)^{\mathbb{N}}$. But the latter is obvious, because any sequence $\left(a_{i}\right) \in\left(\mathbb{Z}_{2}\right)^{\mathbb{N}}$, where $a_{i} \in\{0,1\}$ can be obtained as the image of the loop that winds around the $i$-th circle exactly when $a_{i}=1$.

Example 3.11. We are going to show that a minor modification of covering maps in the inverse sequence described in example 3.7 yields a completely different limit space. This is surprising and very difficult to see geometrically. Let us define two covering projections with fibre $\mathbb{Z}$. The first is the standard covering exponential map from the real line to the circle

$$
e: \mathbb{R} \rightarrow S^{1}, \quad e(t):=e^{2 \pi i t}
$$

and the second combines the exponential covering with the two-fold covering of the circle given by the squaring map

$$
f: \mathbb{R} \times S^{1} \rightarrow S^{1} \times S^{1}, \quad f(t, z):=\left(e^{2 \pi i t}+z^{2}, z\right),
$$

Note that the induced homomorphism $f_{*}: \pi_{1}\left(\mathbb{R} \times S^{1}\right) \rightarrow \pi_{1}\left(S^{1} \times S^{1}\right)$ sends the generator of $\pi_{1}\left(\mathbb{R} \times S^{1}\right) \cong \mathbb{Z}$ to the element $(2,1) \in \pi_{1}\left(S^{1} \times S^{1}\right) \cong \mathbb{Z} \oplus \mathbb{Z}$, therefore $f$ can be characterized as the covering of $S^{1} \times S^{1}$ corresponding to the cyclic subgroup of $\mathbb{Z} \times \mathbb{Z}$ generated by the element $(2,1)$.

The following diagram depicts two inverse sequences of covering maps over $\left(S^{1}\right)^{\mathbb{N}}$.

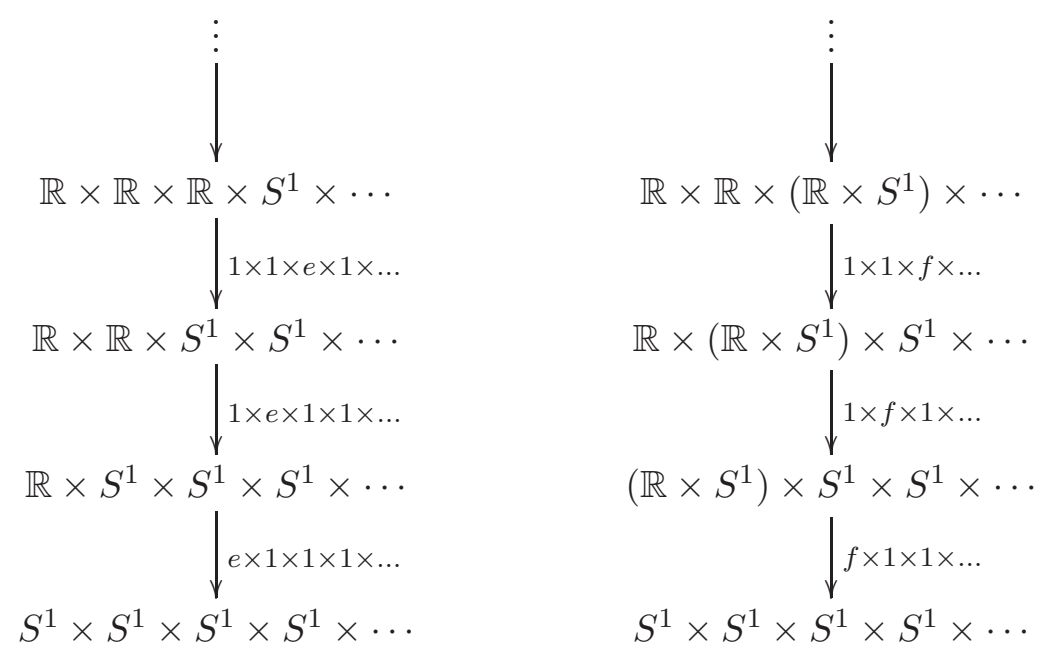


The first sequence was already considered in Example 3.7 where we showed that its inverse limit is $\mathbb{R}^{\mathbb{N}}$, which is, of course, path-connected. The second is also a sequence of $\mathbb{Z}$-covering maps and the form of the total spaces suggest that its limit is $\mathbb{R}^{\mathbb{N}}$ as well. However, it is not difficult to check that there does not exist a map $\mathbb{R}^{\mathbb{N}} \rightarrow\left(S^{1}\right)^{\mathbb{N}}$ that factors through all terms in the sequence. Thus, the question is what is the inverse limit of the second sequence of coverings?

To compute the set of path components of the inverse limit of the second sequence we compare it with the following sequence over a polyhedral expansion of $\left(S^{1}\right)^{\mathbb{N}}$ :

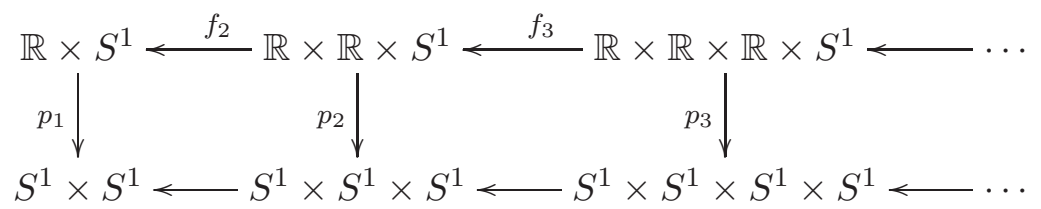

The maps $f_{i}: \mathbb{R}^{i} \times S^{1} \rightarrow \mathbb{R}^{i-1} \times S^{1}$ are defined as a product of a projection on the first $i$-components with the square map (i.e. two-fold covering map) on $S^{1}$. Furthermore, the maps $p_{i}: \mathbb{R}^{i} \times S^{1} \rightarrow\left(S^{1}\right)^{i+1}$ are covering maps corresponding to respectively the cyclic subgroup generated by the element $\left(2^{i}, 2^{i-1}, \ldots, 2,1\right) \in \mathbb{Z}^{i+1}=\pi_{1}\left(\left(S^{1}\right)^{i+1}\right)$. It is easy to check that the diagram commutes and that the $i$-th term in the original sequence is the pull-back of $p_{i}$ along the projection map as in the diagram

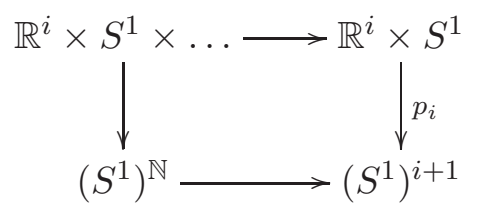

It follows that the inverse limit of the original sequence and the inverse limit of the sequence of covering over the polyhedral expansion coincide. We may now apply Theorem 3.5 to determine the set of components of the total space of the limit. In fact, the inverse sequence of the fundamental groups of the covering spaces is

$$
\mathbb{Z} \stackrel{2}{2}^{2} \stackrel{2}{\longleftarrow} \mathbb{Z} \stackrel{2}{\longleftarrow} \mathbb{Z} \longleftarrow \cdots
$$

which is not Mittag-Leffler, therefore its derived inverse limit is non-trivial (it is actually an uncountable abelian group). As a consequence, the total space of the inverse limit has uncountably many components. Indeed, by a closer examination of the inverse limit of coverings over the polyhedral expansion of $\left(S^{1}\right)^{\mathbb{N}}$ we can conclude that the total space of the limit in the second case is homeomorphic to the product of the dyadic solenoid $\mathrm{Sol}_{2}$ with $\mathbb{R}^{\mathbb{N}}$. 


\section{Algebraic applications}

We begin by describing a construction of inverse sequences of covering projections whose limits correspond to meaningful subgroups of the fundamental group. Given a countable CW-complex $X$ and a continuous map $f: X \rightarrow S^{1}$ let $p: \widetilde{X} \rightarrow X$ be the covering map obtained as a pullback of the universal covering of $S^{1}$ along $f$, as in the following diagram

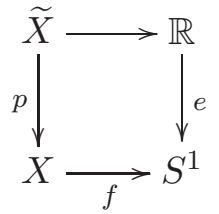

It is easy to check that $p$ is the covering map that corresponds to the kernel of $f_{*}$, that is to say

$$
\pi_{1}(\tilde{X}) \cong \operatorname{Im}\left(p_{*}: \pi_{1}(\tilde{X}) \rightarrow \pi_{1}(X)\right)=\operatorname{Ker}\left(f_{*}: \pi_{1}(X) \rightarrow \pi_{1}\left(S^{1}\right)\right) .
$$

Clearly, $p$ is a regular covering whose fibres can be naturally identified with the infinite cyclic group $\mathbb{Z}$, so we often say that $p$ is the $\mathbb{Z}$-covering, corresponding to the map $f: X \rightarrow S^{1}$ (or rather, to its homotopy class). Since $S^{1}$ is an Eilenberg-MacLane space of type $K(\mathbb{Z}, 1)$, there is a bijection

$$
\left[X, S^{1}\right]=\operatorname{Hom}\left(\pi_{1}(X), \mathbb{Z}\right) .
$$

Thus, we may also say that $p$ is the $\mathbb{Z}$-covering corresponding to a given homomorphism $\varphi: \pi_{1}(X) \rightarrow \mathbb{Z}$, in the sense that $\pi_{1}(\widetilde{X})=\operatorname{Ker} \varphi$. Note, that every non-trivial subgroup of $\mathbb{Z}$ is isomorphic to $\mathbb{Z}$, so we may assume without loss of generality that $\varphi$ is surjective.

Given a sequence of homomorphisms $\varphi_{1}, \varphi_{2}, \varphi_{3}, \ldots: \pi_{1}(X) \rightarrow \mathbb{Z}$, let

$$
\Phi_{n}:=\left(\varphi_{1}, \ldots, \varphi_{n}\right): \pi_{1}(X) \rightarrow \mathbb{Z}^{n}
$$

and let $p_{n}: \widetilde{X}_{n} \rightarrow X$ be the covering projection whose fundamental group is

$$
K_{n}:=\operatorname{Ker} \Phi_{n}=\operatorname{Ker} \varphi_{1} \cap \ldots \cap \operatorname{Ker} \varphi_{n} .
$$

Thus we obtain an inverse sequence of coverings

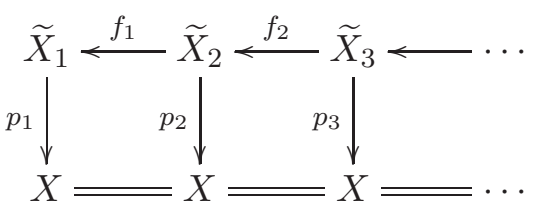

where each $f_{n}$ is a covering projection. To compute the fibre of $f_{n}$ note that have a short exact sequence

$$
0 \rightarrow K_{n-1} / K_{n} \longrightarrow \pi_{1}(X) / K_{n} \longrightarrow \pi_{1}(X) / K_{n-1} \rightarrow 0
$$

where the second and third group are subgroups of $\mathbb{Z}^{n}$ and $\mathbb{Z}^{n-1}$ respectively. From this we deduce that $K_{n-1} / K_{n}$ is either trivial or isomorphic to $\mathbb{Z}$, therefore all $f_{n}$ are either trivial coverings (identity maps) or $\mathbb{Z}$-coverings. 
Since $\operatorname{Hom}\left(\pi_{1}(X), \mathbb{Z}\right)$ is countable, we may apply the above construction to the sequence of all homomorphisms from $\pi_{1}(X)$ to $\mathbb{Z}$. The limit of the resulting inverse sequence of coverings is a lifting projection $\widehat{p}: \widehat{X} \rightarrow X$ whose fundamental group is

$$
\pi_{1}(\widehat{X})=\bigcap_{\varphi: \pi_{1}(X) \rightarrow \mathbb{Z}} \operatorname{Ker} \varphi
$$

Note that if $\pi_{1}(X)$ is finitely generated (e.g., if the 1-skeleton of $X$ is finite), then $\widehat{p}$ is a covering projection. In fact, in that case $\operatorname{Hom}\left(\pi_{1}(X), \mathbb{Z}\right)=$ $\operatorname{Hom}\left(H_{1}(X), \mathbb{Z}\right)$ is also finitely generated and the tower of coverings is actually finite (i.e., all but finitely many coverings in the sequence are trivial). Alternatively, $\widehat{p}$ can be obtained as a covering of $X$ that corresponds to the kernel of the natural homomorphism $\pi_{1}(X) \rightarrow F H_{1}(X)$, where $F H_{1}(X)$ denotes the maximal free abelian quotient of $H_{1}(X)$.

What is the algebraic meaning of the intersection of kernels of homomorphisms to some group? It is well-known that the commutator subgroup $F_{n}^{\prime}=\left[F_{n}, F_{n}\right]$ of the free group on $n$ generators $F_{n}$ consists of all words in $F_{n}$ for which the sum of exponents of each letter equals 0 . This description is not intrinsic, as it requires to choose a basis for the free group. An equivalent description without reference to a basis is the following: if $F$ is a free group (on any set of generators), then

$$
F^{\prime}=\bigcap_{f: F \rightarrow \mathbb{Z}} \operatorname{Ker}(f)
$$

This approach was used in Cannon-Conner [2, Section 4] to describe the big commutator subgroup of the fundamental group of the Hawaiian earring $\mathcal{G}=\pi_{1}(H)$ :

$$
B C(\mathcal{G})=\bigcap_{f: \mathcal{G} \rightarrow \mathbb{Z}} \operatorname{Ker}(f) .
$$

The big commutator subgroup is much larger than the usual commutator subgroup $\mathcal{G}^{\prime}$ but shares many interesting properties with the latter.

One can consider intersections of homomorphisms to other groups as well, for example finite or torsion-free groups. In [11] we gave an intrinsic description of he second commutator subgroup $F_{n}^{\prime \prime}$ using homomorphisms to the Baumslag-Solitar group $B(1, n)$.

Let us introduce the following functor for arbitrary groups $G$ and $A$ :

$$
\operatorname{Ker}_{A}(G):=\bigcap_{f: G \rightarrow A} \operatorname{Ker}(f) .
$$

Note that $\operatorname{Ker}_{A}(G)$ is a fully characteristic subgroup of $G$. More generally, if $\varphi: G \rightarrow H$ is a homomorphism and if $x \in \operatorname{Ker}_{A}(G)$, then for every $\psi: H \rightarrow$ $A$ we have that $\psi \circ \varphi(x)=0$, therefore $\varphi(x) \in \operatorname{Ker}_{A}(H)$. In categorical terms the correspondence $G \mapsto \operatorname{Ker}_{A}(G)$ is a covariant functor. It turns 
out that many characteristic subgroups can be described as intersection of kernels to some group $A$. In fact we may view $\operatorname{Ker}_{A}(G)$ as the part of $G$ that cannot be represented in a product of copies of the group $A$. For example $\operatorname{Ker}_{\mathbb{Z}}(G)=0$ if, and only if, $G$ is residually free-abelian.

We mentioned before that $\operatorname{Ker}_{\mathbb{Z}}(F)=F^{\prime}$ and $\operatorname{Ker}_{B(1, n)}(F)=F^{\prime \prime}$ for every free group $F$. Recall that a torsion-free abelian group $A$ is slender if every homomorphism $\varphi: \mathbb{Z}^{\mathbb{N}} \rightarrow A$ can be factored through some finite rank free abelian group, i.e., there exists a homomorphism $\varphi^{\prime}: \mathbb{Z}^{n} \rightarrow A$ so that the following diagram commutes

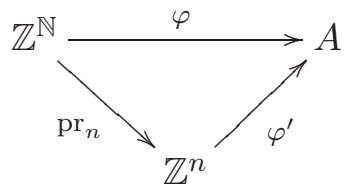

(see Fuchs [16, Ch. XIII]) where $\mathrm{pr}_{n}$ is the projection map onto the first $n$ factors of $\mathbb{Z}^{\mathbb{N}}$. Free abelian groups are slender, subgroups and extensions of slender groups are also slender. A reduced abelian group is slender if, and only if, it does not contain a subgroup isomorphic to the group $\mathbb{Z}^{\mathbb{N}}$ or to a group of $p$-adic integers for some prime $p$ (cf. Fuchs [16]).

Katsuya Eda [14] extended this concept to arbitrary groups by defining a group $A$ to be non-commutatively slender (nc-slender) if every homomorphism $\varphi: \mathcal{G} \rightarrow A$ from the Hawaiian earring group $\mathcal{G}$ to $A$ can be factored through some free (non-commutative) group of finite rank as in

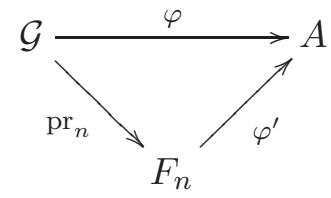

where $\mathrm{pr}_{n}$ is the homomorphism induced by collapsing all but the first $n$ loops of the Hawaiian earring.

Lemma 4.1. If $A$ is slender, then

$$
\operatorname{Ker}_{A}\left(\mathbb{Z}^{\mathbb{N}}\right)=\lim _{\longleftarrow} \operatorname{Ker}_{A}\left(\mathbb{Z}^{n}\right) .
$$

Similarly, if $A$ is nc-slender, then

$$
\operatorname{Ker}_{A}(\widehat{F})=\lim _{\longleftarrow} \operatorname{Ker}_{A}\left(F_{n}\right) \text { and } \operatorname{Ker}_{A}(\mathcal{G})=\mathcal{G} \cap \lim _{\longleftarrow} \operatorname{Ker}_{A}\left(F_{n}\right) \text {. }
$$

Proof. The natural projections $\operatorname{pr}_{n}: \mathbb{Z}^{\mathbb{N}} \rightarrow \mathbb{Z}^{n}$ induce an isomorphism $\mathbb{Z}^{\mathbb{N}} \rightarrow$

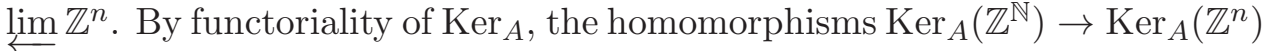
induced by $\operatorname{pr}_{n}$ are coherent and induce a homomorphism $\operatorname{Ker}_{A}\left(\mathbb{Z}^{\mathbb{N}}\right) \rightarrow$ $\lim \operatorname{Ker}_{A}\left(\mathbb{Z}^{n}\right)$. This homomorphism is injective, because it is a restriction of the isomorphism $\mathbb{Z}^{\mathbb{N}} \rightarrow \lim \mathbb{Z}^{n}$. To prove surjectivity, consider an element $\left(x_{i}\right) \in \lim _{\longleftarrow} \operatorname{Ker}_{A}\left(\mathbb{Z}^{n}\right)=\overleftarrow{\mathbb{Z}^{\mathbb{N}}}$. We need only show that $\left(x_{i}\right) \in \operatorname{Ker}_{A}\left(\mathbb{Z}^{\mathbb{N}}\right)$. 
Fix a homorphism $\varphi: \mathbb{Z}^{\mathbb{N}} \rightarrow A$. Since $A$ is slender, $\varphi$ can be factored as $\varphi=\varphi^{\prime} \circ \operatorname{pr}_{n}$ for some $n$ and some $\varphi^{\prime}: \mathbb{Z}^{n} \rightarrow A$. Then $\varphi\left(\left(x_{i}\right)\right)=\varphi^{\prime}\left(x_{n}\right)$ which is trivial since $x_{n} \in \operatorname{Ker}_{A}\left(\mathbb{Z}^{n}\right)$. Thus $\operatorname{Ker}_{A}\left(\mathbb{Z}^{\mathbb{N}}\right) \rightarrow \underset{\longleftarrow}{\lim } \operatorname{Ker}_{A}\left(\mathbb{Z}^{n}\right)$ is surjective as well.

In [13, the authors show that if $A$ is nc-slender then every homomorphism from $\widehat{F}$ to $A$ also factors through a projection to $F_{n}$ for some $n$. Then the proof for the non-commutatively slender cases follows analogously.

As a consequence, $A$-kernels of $\widehat{F}$ with respect to a slender group $A$ give rise to inverse limits of coverings over the Hawaiian earring. Indeed, recall that the Hawaiian earring $H$ can be represented as inverse limit of a sequence

$$
\cdots \leftarrow X_{n} \leftarrow X_{n+1} \leftarrow \cdots \leftarrow H,
$$

where $X_{n}$ is a wedge of $n$ circles and the bonding maps are the obvious projections. For each $n$ the fundamental group of $X_{n}$ is $F_{n}$, the free group on $n$ generators and there is the universal covering projection $q_{n}: \widehat{X}_{n} \rightarrow$ $X_{n}=\widehat{X}_{n} / F_{n}$. The limit of the resulting inverse sequence of coverings is a fibration with unique path lifting property

$$
q: \widehat{H}=\lim _{\longleftarrow} \widehat{X}_{n} \rightarrow H=\lim _{\longleftarrow} X_{n} .
$$

By [8, Section 4] the group $\widehat{F}$ acts freely and transitively on the fibres of $q$ so we may consider the quotient of $\widehat{H}$ with respect to any (normal) subgroup of $\widehat{F}$.

Proposition 4.2. If $A$ is any nc-slender group, then the projection

$$
q_{A}: \widehat{H} / \operatorname{Ker}_{A}(\widehat{F}) \rightarrow H
$$

can be obtained as the inverse limit of coverings $q_{n}^{\prime}: \widehat{X}_{n} / \operatorname{Ker}_{A}\left(F_{n}\right) \rightarrow X_{n}$, and is therefore a fibration with unique path-lifting property.

Proof. For each $n$ we have a commutative diagram of the form

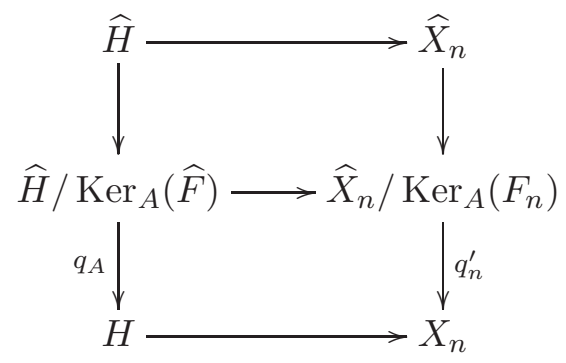

where for each $n$ the map $q_{n}^{\prime}$ is a covering projection with fibre $F_{n} / \operatorname{Ker}_{A}\left(F_{n}\right)$. These coverings form an inverse sequence and by naturality we get a mapping 
to the inverse limit

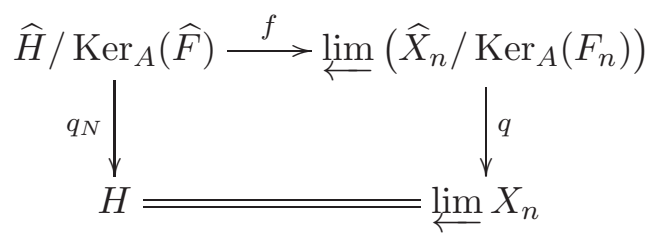

To prove our claim it is sufficient to show that the restriction of $f$ to the fibres

$$
f: \widehat{F} / \operatorname{Ker}_{A}(\widehat{F}) \rightarrow \lim _{\longleftarrow}\left(F_{n} / \operatorname{Ker}_{A}\left(F_{n}\right)\right)
$$

is an isomorphism. Consider the following inverse sequence of short exact sequences

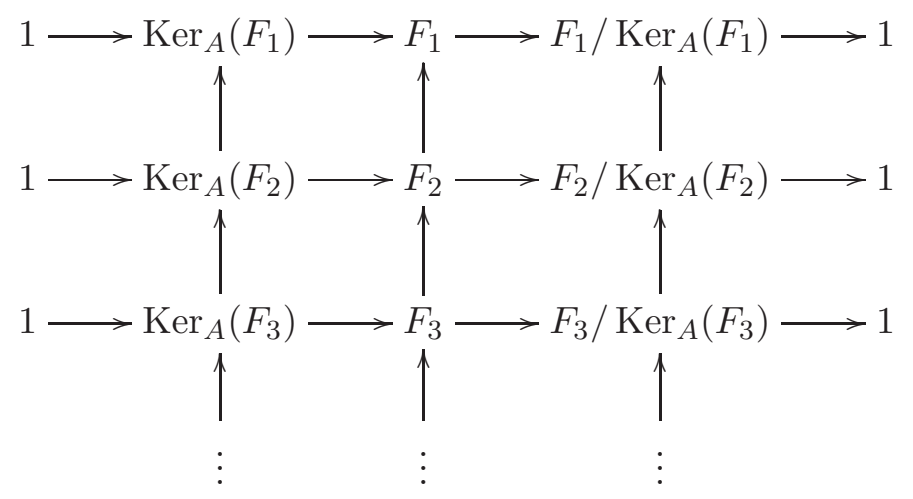

Each projection $F_{n+1} \rightarrow F_{n}$ is a split surjection, so by functoriality of $\operatorname{Ker}_{A}$ all bonding homomorphisms in the first column are surjective. By [19, Section 11.3] the resulting sequence of inverse limits is also exact. Moreover, by Lemma $4.1 \lim _{\leftarrow} \operatorname{Ker}_{A}\left(F_{n}\right)=\operatorname{Ker}_{A} \widehat{F}$ so we have the following short exact sequence

$$
1 \longrightarrow \operatorname{Ker}_{A}(\widehat{F}) \longrightarrow \widehat{F} \longrightarrow \lim _{\longleftarrow}\left(F_{n} / \operatorname{Ker}_{A}\left(F_{n}\right)\right) \longrightarrow 1
$$

which implies that the projections $\widehat{F} \rightarrow F_{n}$ induce the isomorphism

$$
\widehat{F} / \operatorname{Ker}_{A}(\widehat{F}) \cong \lim _{\longleftarrow}\left(F_{n} / \operatorname{Ker}_{A}\left(F_{n}\right)\right)
$$

as claimed.

In order to prove the main results of this section we will need an algebraic lemma that is reminiscent of Theorem 3.5. Let $G$ be a subgroup of the inverse limit of a sequence of groups $\left\{G_{i}\right\}$ such that all projections $G \rightarrow G_{i}$ are surjective. Furthermore, let $H_{i}$ be a decreasing sequence of subgroups of $G$ and for each $i$ let $\bar{H}_{i}$ be the image of $H_{i}$ in $G_{i}$. Observe that $G / H_{i} \cong$ $G_{i} / \bar{H}_{i}$ (a bijection for arbitrary groups $H_{i}$, and a group isomorphism if $H_{i}$ 
are normal subgroups of $G$ ). We can fit the above data in a commutative diagram with exact rows:

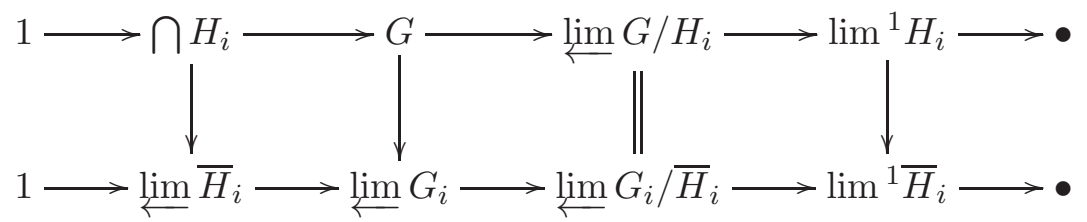

The following lemma is proved by elementary diagram chasing.

Lemma 4.3. $\operatorname{Ker}\left(\bigcap H_{i} \rightarrow \underset{\lim }{\longleftarrow} \bar{H}_{i}\right)=\operatorname{Ker}\left(G \rightarrow \underset{\lim }{\longleftarrow} G_{i}\right)$, thus we have an exact sequence

$$
1 \longrightarrow \operatorname{Ker}\left(G \rightarrow \lim _{\longleftarrow} G_{i}\right) \longrightarrow \bigcap H_{i} \longrightarrow \lim _{\longleftarrow} \bar{H}_{i}
$$

Moreover $\lim ^{1} H_{i} \rightarrow \lim ^{1} \bar{H}_{i}$ is a surjection of homogeneous sets whose fibres can be naturally identified with the cokernel of the homomorphism

$$
G \rightarrow\left(\lim _{\longleftarrow} G_{i}\right) /\left(\lim _{\longleftarrow} \bar{H}_{i}\right)
$$

As a consequence, $\lim ^{1} H_{i}$ is trivial iff $\lim ^{1} \bar{H}_{i}$ is trivial and $G \rightarrow\left(\lim _{\longleftarrow} G_{i}\right) /\left(\lim _{\longleftarrow} \bar{H}_{i}\right)$ is surjective.

We have previously considered the inverse sequence of wedges of circles

$$
\cdots \leftarrow X_{n} \leftarrow X_{n+1} \leftarrow \cdots \leftarrow H
$$

converging to the Hawaiian earring. Since $\pi_{1}\left(X_{n}\right)=F_{n}$, the free group on $n$ generators $F_{n}$, the inverse sequence of spaces gives rise to a homomorphism from the Hawaiian earring group $\mathcal{G}:=\pi_{1}(H)$ to the inverse limit of finite rank free groups $\widehat{F}:=\lim F_{n}$. By a result of Higman [21], see also [2] and [15], the homomorphism $\mathcal{G} \rightarrow \widehat{F}$ is injective, so the Hawaiian earring group can be viewed as a subgroup of $\widehat{F}$. It is known that both the group $\mathcal{G}$ and its index in $\widehat{F}$ are uncountable, and that $\mathcal{G}$ can be viewed as a dense subgroup of $\widehat{F}$ with respect to the inverse limit topology on $\widehat{F}$. The following theorem gives a more precise description of the relation between the two groups. The proof is a combination of algebraic and geometric techniques developed in the previous sections.

Theorem 4.4. The group $\widehat{F}$ is equal to the internal product of its subgroups $\mathcal{G}$ and $\operatorname{Ker}_{\mathbb{Z}}(\widehat{F})$, i.e., $\widehat{F}=\mathcal{G} \cdot \operatorname{Ker}_{\mathbb{Z}}(\widehat{F})$.

Proof. We are going to apply Lemma 4.3 to the following data: let $G_{i}$ be the free group on $i$ generators $F_{i}, G=\mathcal{G}$, and $H_{i}$ the kernel of the epimorphism $\mathcal{G} \rightarrow \pi_{1}\left(X_{i}\right) \rightarrow H_{1}\left(X_{i}\right) \cong \mathbb{Z}^{i}$. Then $\lim _{i} G_{i}=\widehat{F}$. Observe that the image of $H_{i}$ in $\pi_{1}\left(X_{i}\right)=F_{i}$ is precisely the commutator subgroup $F_{i}^{\prime}=\operatorname{Ker}_{\mathbb{Z}}\left(F_{i}\right)$, since $\mathcal{G}$ maps surjectively onto $\pi_{1}\left(X_{i}\right)$. The bonding maps in the inverse 
sequence of groups $\left\{\bar{H}_{i}\right\}$ are surjective, therefore $\lim ^{1} \bar{H}_{i}$ is trivial. Moreover, by Lemma $4.1 \underset{\lim }{\longleftarrow} \bar{H}_{i}=\lim \operatorname{Ker}_{\mathbb{Z}}\left(F_{i}\right)=\operatorname{Ker}_{\mathbb{Z}}(\widehat{F})$. Thus, by Lemma 4.3 the formula $\widehat{F}=\widehat{G} \cdot \operatorname{Ker}_{\mathbb{Z}}(\widehat{F})$ holds if, and only if, the derived inverse limit $\lim ^{1} H_{i}$ is trivial.

It is well-known that the limit $\lim ^{1} H_{i}$ is precisely the set of path-components of the inverse limit of covering maps that correspond to the sequence of kernels of the homomorphisms $\left\{\mathcal{G} \rightarrow H_{1}\left(X_{i}\right)\right\}$ (see [4, 22], as well as our discussion in Section 2). Analogously as in the examples in Section 3, we can represent this lifting projection as a limit of covering spaces over the approximations of the Hawaiian earrings by finite wedges of circles $X_{i}$. The covering space over $X_{i}$ corresponding to the commutator subgroup $F_{i}$ can be identified with the integral 1-dimensional grid in $\mathbb{R}^{i}$, i.e.

$$
\widetilde{X}_{i}=\left\{\left(x_{1}, \ldots, x_{i}\right) \in \mathbb{R}^{i} \mid x_{j} \notin \mathbb{Z} \text { for at most one index } j\right\} .
$$

Observe that the bonding maps in the inverse system

$$
\tilde{X}_{1} \longleftarrow \widetilde{X}_{2} \longleftarrow \tilde{X}_{3} \longleftarrow \cdots
$$

are retractions, and so their inverse limit $\tilde{X}$ is the 1-dimensional integral grid in $\mathbb{R}^{\mathbb{N}}$. Alternatively, we may describe $\widetilde{X}$ by the following pullback diagram

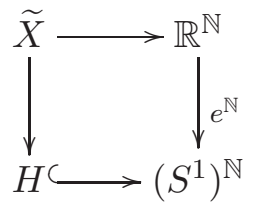

Clearly, $\widetilde{X}$ is path-connected, which completes the proof of our claim.

Note that there one could also consider the commutator subgroup $\widehat{F}^{\prime}$ of $\widehat{F}$, which is however much smaller than $\lim _{n} F_{n}^{\prime}$. In fact, $\widehat{F}>\mathcal{G} \cdot \widehat{F}^{\prime}$.

Continuing the previous line of thought we may ask whether $\widehat{F}$ can be obtained by adding some other term of the derived series of $\widehat{F}$ to the group $\mathcal{G}$. As before, we replace $\widehat{F}^{\prime \prime}$ with a suitable inverse limit group. We already mentioned that the second derived group of a free group $F$ can be described as a kernel, $F^{\prime \prime}=\operatorname{Ker}_{B}(F)$ (see [11, Theorem 1]) for any solvable, deficiency 1 group $B$ that is not virtually abelian. By work of Wilson [31, any solvable deficiency 1 group is isomorphic to a Baumslag-Solitar group $B(1, m)$ for some $m$. Since $B(1, m)$ is nc-slender (see [5]), Lemma 4.1 implies that $\operatorname{Ker}_{B}(\widehat{F})=\lim \operatorname{Ker}_{B}\left(F_{n}\right)$ for every group $B$ that is solvable, of deficiency 1 and is not virtually abelian. As well, we have

$$
\widehat{F}^{\prime \prime} \leq \operatorname{Ker}_{B}(\widehat{F}) \leq \operatorname{Ker}_{\mathbb{Z}}(\widehat{F}) .
$$


In the next theorem we reverse the reasoning and use our methods to show that this subgroup of $\widehat{F}$ is too small to generate, together with the Hawaiian earring group the entire group $\widehat{F}$.

Theorem 4.5. $\widehat{F} \neq \mathcal{G} \cdot \operatorname{Ker}_{B}(\widehat{F})$ for every group $B$ that is solvable, of deficiency 1 and is not virtually abelian.

Proof. Let $B$ be a solvable deficiency 1 group that is not virtually abelian. Then $B$ is isomorphic to a Baumslag-Solitar group $B(1, m)$ and, for a free group of rank $n$, we have $\operatorname{Ker}_{B}\left(F_{n}\right)=F_{n}^{\prime \prime}=\operatorname{Ker}_{\mathbb{Z}}\left(\operatorname{Ker}_{\mathbb{Z}}\left(F_{n}\right)\right)$. We may repeat almost verbatim the algebraic part of the proof of the previous theorem and obtain that $\widehat{F}=\mathcal{G} \cdot \operatorname{Ker}_{B}(\widehat{F})$ if, and only if, the inverse limit $\widetilde{Y}$ of the sequence of coverings over the Hawaiian earring $H$, determined by the kernels of homomorphisms $\mathcal{G} \rightarrow F_{i} / F_{i}^{\prime \prime}$, is path-connected. Thus, in order to prove our claim, we must show that $\tilde{Y}$ is not path-connected.

We will use the same notation as in the proof of the previous theorem. Let $\widetilde{X}$ be the integral grid in $\mathbb{R}^{\mathbb{N}}$ and let $p: \widetilde{X} \rightarrow H$ be the lifting projection obtained as the limit of the sequence

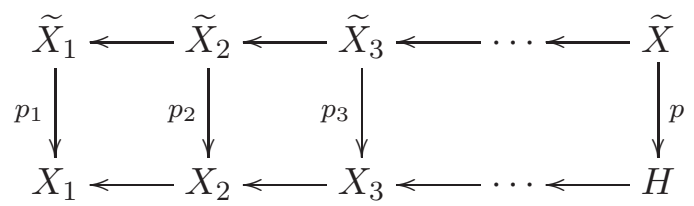

where $\pi_{1}\left(\widetilde{X}_{n}\right)=F_{n}^{\prime}$. Let $\widetilde{Y}_{n}$ be a cover of $\widetilde{X}_{n}$ corresponding to the subgroup $\left(\pi_{1}\left(\widetilde{X}_{n}\right)\right)^{\prime}=F_{n}^{\prime \prime}$ and let $\widetilde{Y}$ be the resulting inverse limit. We will construct a continuous surjection from $\widetilde{Y}$ to a path-disconnected space. Since $\pi_{1}\left(\widetilde{X}_{2}\right)=F_{2}^{\prime}$ is a countably generated free group, we can find a sequence of homomorphisms $f_{i}: \pi_{1}\left(\widetilde{X}_{2}\right) \rightarrow \mathbb{Z}$ such that

$$
\bigcap_{i=1}^{n} \operatorname{Ker}\left(f_{i}\right) \subsetneq \bigcap_{i=1}^{n-1} \operatorname{Ker}\left(f_{i}\right) \text { and } \bigcap_{i=1}^{\infty} \operatorname{Ker}\left(f_{i}\right)=F_{2}^{\prime \prime} \text {. }
$$

Let $\widetilde{Z}_{n}^{\prime}$ be the cover of $\widetilde{X}_{2}$ corresponding to the subgroup $\bigcap_{i=1}^{n} \operatorname{Ker}\left(\pi_{1}\left(\widetilde{X}_{2}\right) \stackrel{f_{i}}{\longrightarrow}\right.$ $\mathbb{Z}$ ). The covers $\widetilde{Z}_{n}^{\prime}$ form an inverse sequence, whose limit $\widetilde{Z}^{\prime}:=\lim _{\longleftarrow} \widetilde{Z}_{n}^{\prime}$ is path-disconnected by Corollary 2.2 . As a consequence, the pullback of $\widetilde{Z}^{\prime}$ along the projection $p: \widetilde{X} \rightarrow \widetilde{X}_{2}$

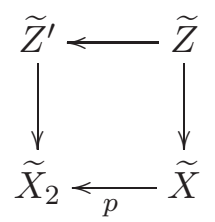


yields a lifting projection $\widetilde{Z} \rightarrow \widetilde{X}$, whose total space is also path-disconnected. Note that $\widetilde{Z}$ can be alternatively obtained as inverse limit of a sequence $\widetilde{Z}=\lim _{\breve{Z}} \widetilde{Z}_{n}$, where $\widetilde{Z}_{n}$ is the pull-back of $\widetilde{Z}_{n}^{\prime}$ along the projection $p_{n}: \widetilde{X}_{n} \rightarrow$ $\widetilde{X}_{2}$. Since commutator subgroups are characteristic we have $p_{n *}\left(\pi_{1}\left(\widetilde{Y}_{n}\right)\right) \leq$ $\pi_{1}\left(\widetilde{Y}_{2}\right) \leq \pi_{1}\left(\widetilde{Z}^{\prime}\right)$, thus $\pi_{1}\left(\widetilde{Y}_{n}\right) \leq \pi_{1}\left(\widetilde{Z}_{n}\right)=p_{n *}^{-1}\left(\pi_{1}\left(\widetilde{Z}^{\prime}\right)\right)$. It follows that $\widetilde{Y}_{n}$ covers $\widetilde{Z}_{n}$ for every $n$ and we obtain the following diagram:

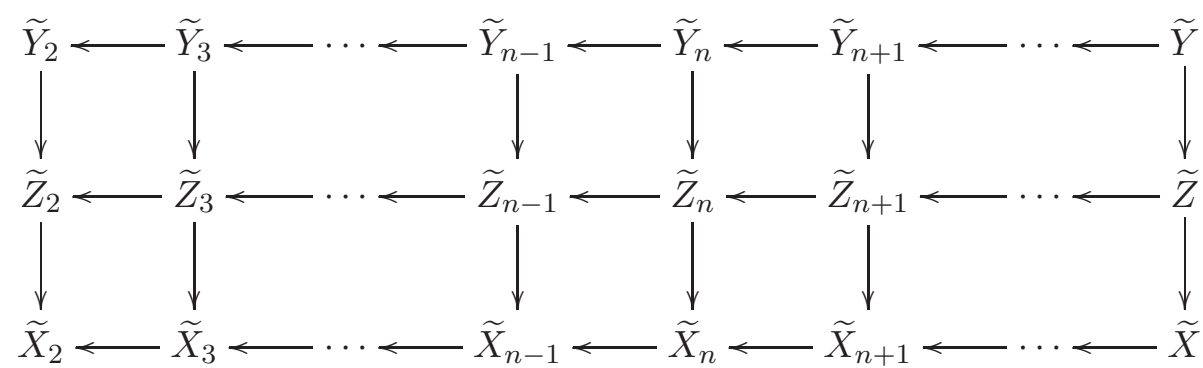

By construction, the fibre of the lifting projection $\widetilde{Y} \rightarrow \widetilde{X}$ maps surjectively to the fibre of $\widetilde{Z} \rightarrow \widetilde{X}$. Since every point in $\widetilde{Z}$ (respectively $\widetilde{Y}$ ) is connected by a path to a point in the fibre, it follows, that the projection $\widetilde{Y} \rightarrow \widetilde{Z}$ is surjective, therefore $\widetilde{Y}$ is not path-connected.

\section{REFERENCES}

[1] A. K. Bousfield and D. M. Kan. Homotopy limits, completions and localizations. Lecture Notes in Mathematics, Vol. 304. Springer-Verlag, Berlin-New York, 1972.

[2] J. Cannon, G. Conner, The combinatorial structure of the Hawaiian earring group, Top. Appl. 106 (2000), 225-271.

[3] J. Cannon, G. Conner, On the fundamental groups of one-dimensional spaces, Top. Appl. 153 (2006), 2648-2672.

[4] Joel M. Cohen. Homotopy groups of inverse limits. In Proceedings of the Advanced Study Institute on Algebraic Topology (Aarhus Univ., Aarhus, 1970), Vol. I, pages 2943. Various Publ. Ser., No. 13, 1970.

[5] Gregory R. Conner and Samuel M. Corson. A note on automatic continuity. Proc. Amer. Math. Soc., 147(3):1255-1268, 2019.

[6] G.R. Conner and K. Eda. Fundamental groups having the whole information of spaces. Topology Appl., 146/147:317-328, 2005.

[7] G. Conner and M. Meilstrup. Deforestation of Peano continua and minimal deformation retracts. Topology Appl., 159(15):3253-3262, 2012.

[8] Gregory R. Conner and Petar Pavešić. General theory of lifting spaces. arXiv e-prints, page arXiv:2008.11267, August 2020.

[9] G. Conner, W. Herfort, P. Pavešić, Some anomalous examples of lifting spaces, Top. Appl. 239 (2018), 234-243.

[10] G. Conner, W. Herfort, P. Pavešić, Geometry of compact lifting spaces, Monatsh Math (2020), https://doi.org/10.1007/s00605-020-01460-1.

[11] G. Conner, C. Kent, W. Herfort, P. Pavešić, Recognizing the Second Derived Group of Free Groups, J. Algebra 516 (2018), 396-400.

[12] G. Conner, C. Kent, W. Herfort, P. Pavešić, Path space and covering fibrations, preprint.

[13] G. Conner, C. Kent, W. Herfort, P. Pavešić, Inverse Limit Slender, preprint. 
[14] Katsuya Eda. Free $\sigma$-products and non-commutatively slender groups. J. Algebra, 148(1):243-263, 1992.

[15] K. Eda and K. Kawamura. The fundamental groups of one-dimensional spaces. Topology and Appl., 87 (1998), 163-172.

[16] L. Fuchs, Infinite abelian groups, vol. II, Pure and Applied Mathematics 36, (Academic Press, Boston, 1973).

[17] Ross Geoghegan, A note on the vanishing of $\lim ^{1}$, J. Pure Appl. Algebra 17 (1980), $113-116$.

[18] Ross Geoghegan and Józef Krasinkiewicz. Empty components in strong shape theory. Topology Appl., 41(3):213-233, 1991.

[19] R. Geoghegan, Topological methods in group theory, Graduate Texts in Mathematics 243 (Springer, New York, 2008)

[20] Masayoshi Hata. On the structure of self-similar sets. Japan J. Appl. Math., 2(2):381414, 1985.

[21] Higman, Graham. Unrestricted free products, and varieties of topological groups. J. London Math. Soc., 27 (1952), 73-81.

[22] Philip S. Hirschhorn. The homotopy groups of the inverse limit of a tower of fibrations. arXiv e-prints, page arXiv:1507.01627, July 2015.

[23] Michael Kapovich and Bruce Kleiner. Hyperbolic groups with low-dimensional boundary. Ann. Sci. École Norm. Sup. (4), 33(5):647-669, 2000.

[24] Józef Krasinkiewicz. Continuous images of continua and 1-movability. Fund. Math. 98 (1978), no. 2, 141-164.

[25] Benoit B. Mandelbrot. On the dynamics of iterated maps. VII. Domain-filling ("Peano") sequences of fractal Julia sets, and an intuitive rationale for the Siegel discs. In Chaos, fractals, and dynamics (Guelph, Ont., 1981/1983), volume 98 of Lecture Notes in Pure and Appl. Math., pages 243-253. Dekker, New York, 1985.

[26] S. Mardešić, J. Segal, Shape Theory, North Holland Mathematical Library, Vol. 26 (North Holland Publishing Company, Amsterdam, 1982).

[27] S. Shelah, Can the fundamental (homotopy) group of a space be the rationals?, Proc. AMS, 103 (1988), 627-632.

[28] E. H. Spanier, Algebraic Topology, (Springer-Verlag, New York 1966).

[29] Peter R. Massopust. Fractal Peano curves. J. Geom., 34(1-2):127-138, 1989.

[30] R. M. Vogt. On the dual of a lemma of Milnor. In Proceedings of the Advanced Study Institute on Algebraic Topology (1970), Vol. III, pages 632-648. Various Publ. Ser., No. 13, 1970.

[31] John S. Wilson. Soluble groups of deficiency 1. Bull. London Math. Soc., 28(5):476480, 1996. 
Department of Mathematics, Brigham Young University, Provo, Ut 84602, USA

Email address: conner@mathematics . byu.edu

Institute for Analysis and Scientific Computation Technische Universität Wien Wiedner Hauptstrasse 8-10/101

Email address: wolfgang.herfort@tuwien.ac.at

Department of Mathematics, Brigham Young University, Provo, Ut 84602, USA

Email address: curtkent@mathematics.byu.edu

Faculty of Mathematics and Physics University of Ljubljana Jadranska 21 LJUbljana, SLOVEnia

Email address: petar.pavesic@fmf.uni-lj.si 